\title{
Deficiência de Vitamina A em mães e filhos no Estado de Pernambuco
}

\author{
Vitamin A deficiency in mothers and children \\ in the state of Pernambuco
}

Teresa Cristina Miglioli ${ }^{1}$

Vania Matos Fonseca ${ }^{1}$

Saint Clair Gomes Junior ${ }^{1}$

Pedro Israel Cabral de Lira ${ }^{2}$

Malaquias Batista Filho ${ }^{3}$

${ }^{1}$ Instituto Fernandes Figueira, Fiocruz. Avenida Rui Barbosa 716, Flamengo. 22250-020 Rio de Janeiro

RJ.tcmiglioli@terra.com.br ${ }^{2}$ Departamento de Nutrição,

Universidade Federal de Pernambuco.

${ }^{3}$ Instituto de Medicina

Integral Professor Fernando Figueira, IMIP.
Abstract The prevalence and risk factors associated with vitamin A deficiency (VAD) were analyzed in 664 mothers and their 790 children under the age of five in Pernambuco in 2006. A population-based cross-sectional study, with a representative sample in urban and rural areas was conducted. VAD was defined as serum retinol levels $<$ $20 \mathrm{mg} / \mathrm{dL}$ for both groups (mothers and children). $B i$ and multivariate analysis were performed using the complementary log-log model as a link function adopting a model of explanatory hierarchical order of VAD to children and a logistic model for mothers. The prevalence of VAD was $6.9 \%$ in mothers and $16.1 \%$ in their children, with similar statistics for urban and. rural groups. In the urban area, the housing occupation regime had a significant association with VAD in mothers. After the final adjustment of the model for urban children, the mother's age, the number of pre-natal consultations and birth weight were featured as predictive variables. In rural areas, VAD in mothers is associated with VAD in children; $P R=$ 3.99 (95\% CI:1.73-9.19), with their birth weight and the incidence of diarrhea in the previous 15 days. VAD continues to be a public health issue in the state of Pernambuco, with marked variations in prevalence and associated factors in mothers and children.

Key words Vitamin A deficiency, Prevalence, Mothers and children, Associated factors
Resumo Analisou-se a prevalência e os fatores associados à deficiência de vitamina A (DVA) em mães (664) e seus filhos (790) menores de cinco anos em Pernambuco/2006. Estudo transversal populacional, com amostra representativa do meio urbano e rural. A DVA foi definida como níveis de retinol sérico $<20 \mu \mathrm{g} / \mathrm{dL}$ para os dois grupos (mães e filhos). Análises bi e multivariadas foram realizadas utilizando o modelo log-log complementar como função de ligação adotando-se modelo de ordenação hierárquica explicativa da DVA para as crianças e modelo logístico para as mães. A prevalência de DVA foi 6,9\% nas mães e 16,1\% em seus filhos, com ocorrências semelhantes para cada grupo na comparação urbano x rural. $\mathrm{Na}$ área urbana, o regime de ocupação da moradia teve relação significante com a DVA nas mães. Para crianças urbanas, após o ajuste final do modelo, figuraram como variáveis preditivas a idade da mãe; $n^{\circ}$ de consultas pré-natais e peso ao nascer. No meio rural a DVA materna se associou à DVA dos filhos $R P=3,99$ (IC:1,73-9,19), ao peso ao nascer e diarreia nos últimos 15 dias. A DVA mantém-se como um problema de saúde pública em Pernambuco, com marcantes diferenças de prevalência e fatores associados em mães e filhos.

Palavras-chave Deficiência de vitamina A, Prevalência, Mães, Crianças, Fatores associados 


\section{Introdução}

Afetando cerca de 210 milhões de crianças menores de cinco anos e mulheres no período de gestação e lactação em todo o mundo ${ }^{1}$, a deficiência de vitamina A (DVA) está elencada entre as três grandes prioridades de carências de micronutrientes, ao lado das anemias e dos distúrbios por deficiência de iodo ${ }^{2,3}$. Em mulheres em idade fértil, a utilização de vitamina A pode diminuir em até $40 \%$ a mortalidade materna ${ }^{4}$. Nas crianças, segundo metanálise envolvendo 43 estudos, observou-se que a suplementação de vitamina $\mathrm{A}$ acarreta redução de $24 \%$ no risco de mortalidade em menores de cinco anos 5 . Ademais a DVA é considerada como a principal causa de cegueira evitável em escala mundial ${ }^{1}$.

Apesar do consenso internacional sobre a importância do problema, a situação epidemiológica da DVA não se acha devidamente conhecida, recorrendo-se, na maioria dos países subdesenvolvidos e em desenvolvimento, à estratégia de lançar mão de dados sobre as condições de vida da população para se obter, estimativamente, uma linha de base para o estabelecimento de objetivos, métodos, avaliações e efetividade dos programas de intervenção. Esta situação dificulta consideravelmente o controle do problema, na medida em que estabelece apriorismos muitas vezes distanciados da realidade. Esse foi o caso do Brasil, ao se utilizar como referencial da DVA estudos localizados em espaços geográficos críticos, como localidades do Nordeste, Norte, os vales do Jequitinhonha (Minas Gerais) e do Ribeira (São Paulo) ${ }^{6,7}$.

Em 2006, o Ministério da Saúde realizou um inquérito nacional (PNDS/2006) ${ }^{8}$ indicando que no país a DVA prevalecia em $17,4 \%$ dos menores de cinco anos e em 12,3\% das mulheres não grávidas em idade reprodutiva.

Ao lado das dúvidas que ainda persistem sobre a real magnitude do problema, outras questões correlatas se impõem, como o caso dos fatores que se associam ao problema e, notadamente, sua ocorrência em relação ao binômio mãe-filho.

É evidente que o inventário analítico do problema representa um aspecto de fundamental importância, na medida em que oferece, pela identificação de variáveis significativas, indicações e evidências para estratégias de intervenção e evolução de suas respostas e tendências temporais, sociais e espaciais. Nesta perspectiva, a análise de fatores relacionados com a ocorrência de DVA em mães e seus filhos, representa uma aborda- gem inovadora, seja por razões epidemiológicas, seja pela lógica operacional de possíveis programas de controle no Brasil, concebidos e desenhados para o atendimento de famílias socialmente vulneráveis. Em outras palavras, o núcleo familiar (objeto do Programa Saúde da Família) $)^{9}$ configurado no binômio mãe-filho, deve expressar um risco relativo mais elevado para o segmento materno-infantil, caracterizando, assim, um grupo de interesse eletivo para a focalização do problema. É esta a lógica que justifica os objetivos e o enfoque metodológico do estudo aqui apresentado.

\section{Método}

O estudo foi realizado com dados da pesquisa "Situação alimentar nutricional e de saúde no Estado de Pernambuco: contexto socioeconômico e de serviços" - III Pesquisa Estadual de Saúde e Nutrição (III PESN/PE) realizada entre 10 de maio e 25 de outubro de 2006. Trata-se de um estudo do tipo transversal, de base populacional, com amostra probabilística representativa do meio urbano e rural e que teve como propósito descrever a condição de saúde e nutrição no grupo materno-infantil. Considerou-se como unidade de estudo a família com crianças menores de 5 anos. Outros detalhes sobre o desenho da amostra encontram-se publicados em Miglioli et al. ${ }^{10}$.

Foram selecionadas crianças de ambos os sexos, entre 6 e 59 meses de idade, que foram submetidas, simultaneamente com outros exames, à dosagem de retinol sérico, juntamente com suas mães biológicas, compondo assim o pareamento amostral mãe-filho, em cada casa sorteada.

As crianças que estavam sob a responsabilidade de avós, tias ou do pai, e aquelas que tiveram processo de adoção foram excluídas por não atenderem ao vínculo biológico de maternidade, adotado restritivamente como condição de interesse específico do estudo. Também foram excluídas as crianças cujas mães se encontravam grávidas ou que não forneceram informação a esse respeito. Em relação à exclusão das gestantes, considerou-se que o processo gravídico faz dessa condição, um grupo diferenciado em relação ao padrão nutricional.

O retinol sérico foi processado no Centro de Investigação em Micronutrientes (CIMICRON) da Universidade Federal da Paraíba e analisado pelo método HPLC (High Pressure Liquid Chromatography), segundo a técnica estabelecida por Furr et al. ${ }^{11}$. O CIMICRON é um laboratório 
credenciado pela OMS como referência para determinação de vitamina A em material biológico. Para indicação da deficiência de vitamina A (variável dependente) nos dois grupos (mães e filhos) foram considerados valores de retinol sérico abaixo de $20 \mu \mathrm{g} / \mathrm{dL}(0,70 \mathrm{~mol} / \mathrm{L})$ segundo critério da Organização Mundial da Saúde ${ }^{12}$.

Como variáveis independentes foram considerados fatores biológicos, ambientais, sociodemográficos, maternos, acesso a serviços de saúde e programas sociais e morbidades.

$\mathrm{Na}$ avaliação do estado antropométrico das crianças, foi utilizado o software Anthro-2007 ${ }^{13}$, sendo classificadas pelo indicador antropométrico peso/idade: baixo peso ( $<-2$ escores $z$ ); peso adequado ( $\geq-2$ escores $\mathrm{z}$ a $<2$ escores $\mathrm{z}$ ); peso elevado ( $\geq 2$ escores $\mathrm{z}$ ) e pelo indicador estatura/ idade: baixa/muito baixa estatura ( $<-2$ escores $\mathrm{z})$ e estatura adequada $(\geq-2 \text { escores } z)^{14}$. Para as mulheres a partir dos 20 anos foi utilizado o Índice de Massa Corporal (IMC) calculado pelo peso em $\mathrm{kg}$ dividido pela altura em metros ao quadrado $\left(\mathrm{kg} / \mathrm{m}^{2}\right)$, por ser este o índice mais utilizado para avaliar o estado antropométrico em estudos epidemiológicos. Adotou-se os limites de corte recomendados pela $\mathrm{OMS}^{15}$ : baixo peso $(<18,5)$, peso adequado $(\geq 18,5 \mathrm{a}<25)$, excesso de peso $(\geq$ 25). Para a classificação do estado nutricional das mães adolescentes (até 19 anos) utilizou-se o IMC/ Idade: baixo peso ( $<-2$ escores $\mathrm{z}$ ); peso adequado ( $\geq-2$ escores $\mathrm{z}$ a 2 escores $\mathrm{z}$ ); peso elevado ( $\geq 2$ escores $z)^{16}$.

A dosagem de hemoglobina foi realizada em fotômetro portátil HemoCue. Para discriminação da anemia foi adotado o ponto de corte recomendado pela OMS, nível de hemoglobina abaixo de $11 \mathrm{~g} / \mathrm{dL}$ e $12 \mathrm{~g} / \mathrm{dL}$, para crianças e mulheres, respectivamente ${ }^{3}$.

A associação dos possíveis fatores preditivos da DVA foi inicialmente avaliada por análises bivariadas, para mulheres e crianças, sendo selecionadas para o modelo multivariado as variáveis que apresentaram nesta análise um valor de $\mathrm{p}<0,20$. As análises multivariadas foram realizadas distintamente para cada um dos grupos: mães e filhos.

Um modelo teórico hierárquico de determinação da DVA foi adotado para as crianças baseado em estudo de Andrade ${ }^{17}$ que utilizou dados da pesquisa estadual realizada em Pernambuco em 1997 (II PESN - PE/1997) (Figura 1). Foram construídos modelos de análise multivariada para a área urbana e para a área rural.

Nível I

Fatores socioeconômicos

- Renda per capita familiar

- Escolaridade materna
Fatores ambientais

- Regime de ocupação da moradia - Serviços de saneamento (água, esgoto, lixo)

Nível II

Fatores maternos

. Idade

. DVA

. Estado nutricional (antropometria)

- No consultas no pré-natal

- Suplementação de vitamina A no pós-parto
Acesso a serviços de saúde e programas sociais

- Visita do agente de saúde

- Cadastro no Programa Saúde Família

- Inscrição no Programa Bolsa Família

Nível III

Fatores relacionados à criança

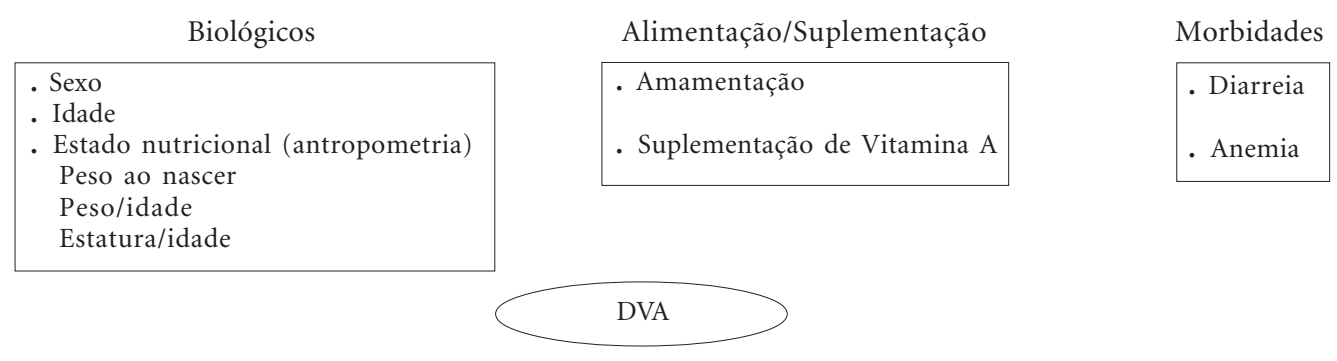

Figura 1. Modelo teórico hierárquico do processo de determinação da deficiência de vitamina A (DVA) em menores de cinco anos. 
As variáveis foram agrupadas em três blocos: no primeiro fizeram parte os fatores socioeconômicos e ambientais (distais); no segundo os fatores relacionados às mães e acesso aos serviços de saúde e programas sociais (intermediários); o terceiro bloco foi composto pelos fatores relacionados às crianças assumidos como proximais (características biológicas, alimentação e morbidades).

Para a construção do modelo final foram introduzidos passo a passo os blocos de variáveis considerando o escalonamento hierárquico distal, intermediário e proximal. Para ajuste do modelo em cada passo permaneceram as variáveis que alcançaram significância de até 0,05

Para as mães foi adotado um modelo hierárquico considerando as variáveis maternas. As análises foram realizadas utilizando o modelo log-log complementar como função de ligação ${ }^{18}$, sendo os resultados expressos por razão de prevalência (RP) e intervalo de confiança (IC) $95 \%$, recorrendo-se ao programa SPSS (Statistical Package for Social Sciences) versão 17.0.

O projeto da III PESN/PE foi aprovado pelo Comitê de Ética e Pesquisa do Instituto de Medicina Integral Prof. Fernando Figueira em 2004.

\section{Resultados}

Do total de 1.650 crianças menores de cinco anos e 1.909 mulheres em idade fértil analisadas na IIIPESN/PE-2006, foram selecionadas 1.454 pessoas que realizaram a dosagem de retinol, sendo 664 mulheres entre 16 e 49 anos e 790 crianças menores de cinco anos. Das mães elegíveis para este estudo, $56(8,4 \%)$ não realizaram os exames laboratoriais, por não comparecerem no momento da coleta de sangue ou por recusa.

Para o total da amostra, níveis inadequados de retinol sérico $(<20 \mu \mathrm{g} / \mathrm{dL})$ foram encontrados em 6,9\% (IC:4,9-8,9) das mães e 16,1\% (IC:13,5-18,6) das crianças. A prevalência de DVA foi de 7,6\% e 6,3\% nas mulheres das áreas urbana e rural, respectivamente. Para as crianças, a ocorrência da DVA na área urbana foi de 14,9\% e na área rural $17,3 \%$.

As análises para relacionar as características biológicas, sociodemográficas e ambientais com a ocorrência de DVA nas mães (Tabela 1) indicaram que, na área urbana, o estado nutricional (IMC) $(\mathrm{p}=0,16)$ e o regime de ocupação da moradia $(\mathrm{p}=0,02)$; e na área rural, o número de consultas no pré-natal $(\mathrm{p}=0,06)$ e suplementação de vitamina A no pós-parto $(\mathrm{p}=0,16)$ foram as variáveis selecionadas para ingressar no modelo logístico, ora adotado, uma vez que, dado o reduzido número de variáveis com significância menor que 0,20 na análise bivariada, não justificava a construção do modelo hierárquico.

Chama a atenção o pequeno número de mães abaixo dos 20 anos de idade, (menos de 10\%), observando-se nesta faixa etária as prevalências menores de DVA, 3,8\% na área urbana e 5,6\% na rural.

A escolaridade das mulheres urbanas foi bem mais elevada: $33 \%$ tinham mais de 9 anos de estudo, enquanto $4,6 \%$ nunca haviam estudado. $\mathrm{Na}$ área rural, apenas 7,9\% estudaram mais de 9 anos e $9,9 \%$ nunca frequentaram a escola. As melhores condições socioeconômicas foram encontradas na área urbana, onde $23 \%$ das mulheres vivem com renda per capita mensal acima de 0,5 salários mínimo, enquanto na área rural a grande maioria (74\%) conta com menos de $1 / 4$ do salário mínimo. Em relação ao número de consultas pré-natais, nas duas áreas, a maior parte das mães realizou seis ou mais consultas, constatando-se que a maior ocorrência de DVA (20\%) foi encontrada no estrato das que não se submeteram a nenhuma consulta.

No caso das mães, na análise multivariada, após o ajuste, o regime de moradia se associou à DVA na área urbana $(\mathrm{p}=0,03) . \mathrm{Na}$ área rural não houve associação significativa da ocorrência de DVA com os fatores analisados.

Em relação à distribuição da DVA nos menores de cinco anos, na análise bivariada para os três blocos do modelo apenas quatro variáveis na área urbana (idade da mãe, número de consulta no pré-natal, peso ao nascer, diarreia nos últimos quinze dias) apresentaram valores de $\mathrm{p}$ $<0,20$, sendo, assim, selecionadas para a construção do modelo multivariado. Para as crianças da área rural, 10 variáveis: escolaridade materna, regime de ocupação da moradia, destino do lixo, DVA da mãe, número de consultas no pré-natal, idade da criança, peso ao nascer, diarreia nas duas últimas semanas anteriores à entrevista, estado nutricional (peso/idade e altura/ idade), foram selecionadas para a análise multivariada (Tabela 2).

No bloco I, os estratos de renda abaixo de 0,5 salário mínimo apresentaram as frequências mais elevadas de DVA. No entanto, esta variável não atingiu o nível de significância proposto para entrada no modelo. No meio rural, as variáveis: escolaridade materna, não ter casa própria $(\mathrm{p}=$ $0,05)$ e lixo não coletado $(p=0,03)$ se associaram com a ocorrência da DVA e foram selecionadas para o modelo multivariado. 
Tabela 1. Prevalência (\%) e razão de prevalência (RP) da deficiência de vitamina A (DVA) em mães de crianças menores de cinco anos, segundo características biológicas, sociodemográficas e ambientais e área de moradia. Pernambuco, 2006.

\begin{tabular}{|c|c|c|c|c|c|c|c|c|}
\hline \multirow[b]{2}{*}{ Variáveis analisadas } & \multicolumn{4}{|c|}{ Área urbana } & \multicolumn{4}{|c|}{ Área rural } \\
\hline & $\begin{array}{c}\text { Amostra } \\
\mathrm{n}=343^{*}\end{array}$ & DVA\% & RP (IC95\%) & $\mathrm{p}$ valor & $\begin{array}{l}\text { Amostra } \\
\mathrm{n}=321^{*}\end{array}$ & DVA\% & RP (IC95\%) & $\mathrm{p}$ valor \\
\hline Idade (anos) & & & & 0,45 & & & & 0,90 \\
\hline$<20$ & 26 & 3,8 & $0,49(0,07-3,46)$ & & 18 & 5,6 & $0,88(0,12-6,25)$ & \\
\hline$\geq 20$ & 278 & 7,9 & 1,0 & & 286 & 6,3 & 1,0 & \\
\hline Estado nutricional (IMC) & & & & 0,16 & & & & 0,65 \\
\hline Baixo peso & 18 & 16,7 & $3,60(0,67-17,44)$ & & 12 & 0 & $* * *$ & \\
\hline Peso normal & 152 & 5,3 & 1,0 & & 153 & 5,9 & 1,0 & \\
\hline Excesso de peso & 133 & 9,0 & $1,79(0,65-4,96)$ & & 137 & 6,6 & $1,13(0,40-3,20)$ & \\
\hline Escolaridade (anos) & & & & 0,98 & & & & 0,91 \\
\hline 0 & 14 & 7,1 & $0,89(0,12-6,61)$ & & 30 & 6,7 & $1,60(0,11-49,07)$ & \\
\hline $1-8$ & 189 & 7,4 & $0,93(0,40-2,13)$ & & 249 & 6,4 & $1,54(0,15-16,61)$ & \\
\hline$\geq 9$ & 100 & 8,0 & 1,0 & & 24 & 4,2 & 1,0 & \\
\hline $\begin{array}{l}\text { Renda per capita familiar } \\
\text { (salário mínimo) }\end{array}$ & & & & 0,79 & & & & 0,82 \\
\hline$<0,25$ & 115 & 8,7 & $1,48(0,48-4,53)$ & & 222 & 5,9 & $1,00(0,14-7,16)$ & \\
\hline $0,25 \vdash 0,50$ & 116 & 7,8 & $1,32(0,42-4,12)$ & & 62 & 8,1 & $1,37(0,17-10,96)$ & \\
\hline$>0,50$ & 68 & 5,9 & 1,0 & & 17 & 5,9 & 1,0 & \\
\hline $\begin{array}{l}\text { Número de pessoas por } \\
\text { cômodo }\end{array}$ & & & & 0,30 & & & & 0,80 \\
\hline$<1$ & 142 & 6,3 & 1,0 & & 117 & 5,1 & 1,0 & \\
\hline $1 \vdash 2$ & 129 & 10,1 & $1,59(0,70-3,59)$ & & 155 & 7,1 & $1,38(0,53-3,63)$ & \\
\hline$\geq 2$ & 33 & 3,0 & $0,48(0,06-3,64)$ & & 32 & 6,3 & $1,22(0,26-5,75)$ & \\
\hline $\begin{array}{l}\text { Regime de ocupação da } \\
\text { moradia }\end{array}$ & & & & 0,02 & & & & 0,23 \\
\hline Casa própria & 194 & 10,3 & 1,0 & & 239 & 7,1 & 1,0 & \\
\hline $\begin{array}{l}\text { Casa alugada/cedida/ } \\
\text { invadida/outro }\end{array}$ & 110 & 2,7 & $0,26(0,08-0,87)$ & & 65 & 3,1 & $0,43(0,10-1,82)$ & \\
\hline Abastecimento de água & & & & 0,33 & & & & 0,29 \\
\hline Rede Geral & 293 & 7,8 & 1,0 & & 40 & 10,0 & 1,0 & \\
\hline Outro & 11 & 0 & $* * *$ & & 264 & 4,5 & $0,57(0,20-1,63)$ & \\
\hline Esgotamento sanitário & & & & 0,48 & & & & 0,72 \\
\hline Rede geral & 201 & 8,0 & 1,0 & & 4 & 0 & 1,0 & \\
\hline Fossa com tampa & 66 & 4,5 & $0,57(0,17-1,90)$ & & 115 & 5,2 & $* * *$ & \\
\hline Outro & 37 & 10,8 & $1,36(0,48-3,83)$ & & 185 & 7,0 & $* * *$ & \\
\hline Destino do lixo & & & & 0,33 & & & & 0,24 \\
\hline Coleta pública & 293 & 7,8 & 1,0 & & 44 & 2,3 & 1,0 & \\
\hline Outra forma & 11 & 0 & $* * *$ & & 260 & 6,9 & $3,05(0,42-22,24)$ & \\
\hline No de consultas no pré-natal & & & & 0,48 & & & & 0,06 \\
\hline Não fez & 5 & 20,0 & $2,59(0,42-15,79)$ & & 9 & 22,2 & $5,14(1,27-20,79)$ & \\
\hline $1-5$ & 55 & 5,5 & $0,71(0,22-2,31)$ & & 97 & 7,2 & $1,67(0,62-4,47)$ & \\
\hline$\geq 6$ & 233 & 7,7 & 1,0 & & 185 & 4,3 & 1,0 & \\
\hline $\begin{array}{l}\text { Suplementação Vitamina A } \\
\text { no pós-parto }\end{array}$ & & & & 0,64 & & & & 0,16 \\
\hline Sim & 15 & 13,3 & 1,0 & & 21 & 4,8 & 1,0 & \\
\hline Não & 33 & 6,1 & $* * *$ & & 27 & 14,8 & $3,11(0,37-25,81)$ & \\
\hline $\begin{array}{l}\text { Não teve filhos nos } \\
\text { últimos } 12 \text { meses }\end{array}$ & 252 & 7,1 & $* * *$ & & 255 & 5,5 & $1,15(0,16-8,35)$ & \\
\hline Visita do agente de saúde & & & & 0,50 & & & & 0,66 \\
\hline Sim (PSF/PACS) & 234 & 8,1 & 1,0 & & 265 & 6,0 & 1,0 & \\
\hline Não & 70 & 5,7 & $0,70(0,25-2,00)$ & & 38 & 7,9 & $1,59(0,48-5,33)$ & \\
\hline Cadastro no PSF & & & & 0,50 & & & & 0,85 \\
\hline Sim & 166 & 6,6 & 1,0 & & 214 & 6,1 & 1,0 & \\
\hline Não & 138 & 8,7 & $1,31(0,60-2,88)$ & & 90 & 6,7 & $1,10(0,43-2,80)$ & \\
\hline Inscrição no PBF & & & & 0,74 & & & & 0,49 \\
\hline Sim & 202 & 7,9 & 1,0 & & 257 & 5,8 & 1,0 & \\
\hline Não & 102 & 6,9 & $0,87(0,37-2,04)$ & & 47 & 8,5 & $1,46(0,51-4,20)$ & \\
\hline
\end{tabular}

"Os totais parciais de cada grupo podem diferir do total da amostra em função de informações ignoradas pelas entrevistadas. " Salário mínimo na época: $\mathrm{R} \$ 350,00 .{ }^{* * *}$ Não pôde ser obtida devido à frequência zero na categoria correspondente 
Do bloco II, as maiores ocorrências de DVA no meio urbano foram encontradas entre os filhos de mães mais jovens ( $\mathrm{RP}=2,13$; IC:1,16$3,90)$ e que não realizaram consultas durante o pré-natal ( $\mathrm{RP}=2,96$; IC:1,36-6,45). $\mathrm{Na}$ área rural, a ocorrência de DVA nas crianças cujas mães também apresentavam a deficiência foi de $38,1 \%$, enquanto entre os filhos de mães sem o problema a prevalência foi $15,2 \%(\mathrm{p}=0,01)$.

Em referência à faixa etária, os resultados evidenciaram menor prevalência de DVA com o aumento da idade das crianças. $\mathrm{Na}$ área rural, nas crianças abaixo de 24 meses, a ocorrência de DVA foi de $21,8 \%$, enquanto nos maiores de 24 meses foi $15,2 \%$. O risco de DVA foi mais elevado nas crianças com quadro diarreico no dia da entrevista ou nas duas semanas anteriores, tanto para as residentes na área urbana $(\mathrm{RP}=1,59$;
IC:0,91-2,75) quanto as da área rural $(\mathrm{RP}=1,75$; IC:1,11-2,79).

Em relação aos indicadores antropométricos (peso/idade, altura/idade), apenas as crianças da área rural apresentaram ambos os indicadores com $\mathrm{p}<0,20$, sendo, portanto, selecionadas para análise multivariada.

A Tabela 3 apresenta as razões de prevalência bruta e ajustada entre a DVA nas crianças da área urbana e os grupos de fatores associados. As duas variáveis que compunham o bloco dos fatores maternos (idade da mãe $[\mathrm{p}=0,004]$ e número de consultas pré-natais: $\mathrm{p}=0,006)$, mantiveram a significância.

Ao incorporar as variáveis do bloco III observa-se que apenas o peso ao nascer $(p=0,01)$ figurou como variável explicativa após o ajuste final do modelo.

Tabela 2. Prevalência (\%) e razão de prevalência (RP) da deficiência de vitamina A (DVA) e associação com variáveis hierarquizadas em crianças menores de cinco anos, segundo área geográfica. Pernambuco, 2006.

\begin{tabular}{|c|c|c|c|c|c|c|c|c|}
\hline \multirow[b]{2}{*}{ Blocos/Variáveis } & \multicolumn{4}{|c|}{ Área urbana } & \multicolumn{4}{|c|}{ Área rural } \\
\hline & $\begin{array}{r}\text { Amostra } \\
\mathbf{n}=397^{*}\end{array}$ & DVA $\%$ & RP (IC95\%) & p valor & $\begin{array}{c}\text { Amostra } \\
\mathbf{n}=393^{*}\end{array}$ & DVA\% & RP (IC95\%) & p valor \\
\hline \multicolumn{9}{|l|}{ Bloco I } \\
\hline \multicolumn{9}{|l|}{ Fatores socioeconômicos } \\
\hline $\begin{array}{l}\text { Renda per capita familiar } \\
\text { (salário mínimo) })^{* *}\end{array}$ & & & & 0,28 & & & & 0,39 \\
\hline$<0,25$ & 155 & 18,1 & $1,69(0,84-3,40)$ & & 301 & 17,9 & $3,23(0,47-22,03)$ & \\
\hline $0,26-0,50$ & 152 & 13,8 & $1,29(0,62-2,69)$ & & 70 & 18,6 & $3,34(0,47-23,90)$ & \\
\hline$>0,50$ & 84 & 10,7 & 1,0 & & 18 & 5,6 & 1,0 & \\
\hline Escolaridade materna (ano & & & & 0,65 & & & & 0,14 \\
\hline 0 & 22 & 18,2 & $1,40(0,54-4,01)$ & & 42 & 21,4 & $0,72(0,32-1,64)$ & \\
\hline $1-8$ & 242 & 15,3 & $1,24(0,72-2,15)$ & & 323 & 15,8 & $0,53(0,28-1,00)$ & \\
\hline$\geq 9$ & 130 & 12,3 & 1,0 & & 27 & 29,6 & 1,0 & \\
\hline \multicolumn{9}{|l|}{ Fatores ambientais } \\
\hline $\begin{array}{l}\text { Regime de ocupação da } \\
\text { moradia }\end{array}$ & & & & 0,69 & & & & 0,05 \\
\hline Casa própria & 258 & 14,3 & 1,0 & & 301 & 15,3 & 1,0 & \\
\hline $\begin{array}{l}\text { Casa alugada/cedida/ } \\
\text { invadida/outro }\end{array}$ & 139 & 15,8 & $1,10(0,68-1,79)$ & & 92 & 23,9 & $1,56(1,00-2,46)$ & \\
\hline Abastecimento de água & & & & 0,36 & & & & 0,59 \\
\hline Rede Geral & 382 & 15,2 & 1,0 & & 48 & 14,6 & 1,0 & \\
\hline Outro & 15 & 6,7 & $0,45(0,07-3,02)$ & & 345 & 17,7 & $1,21(0,59-2,49)$ & \\
\hline Esgotamento sanitário & & & & 0,91 & & & & 0,20 \\
\hline Rede geral & 257 & 15,2 & 1,0 & & 4 & 50,0 & 1,0 & \\
\hline Fossa com tampa & 89 & 13,5 & $0,89(0,49-1,62)$ & & 139 & 18,0 & $0,36(0,13-1,02)$ & \\
\hline Outro & 51 & 15,7 & $1,03(0,51-2,08)$ & & 250 & 16,4 & $0,33(0,12-0,91)$ & \\
\hline Destino do lixo & & & & 0,95 & & & & 0,03 \\
\hline Coleta pública & 383 & 14,9 & 1,0 & & 57 & 7,0 & 1,0 & \\
\hline Outra forma & 14 & 14,3 & $0,96(0,26-3,54)$ & & 336 & 19,0 & $2,71(1,03-7,16)$ & \\
\hline
\end{tabular}


As razões de prevalência bruta e ajustada entre a DVA nas crianças da área rural e os grupos de fatores associados encontram-se na Tabela 4, evidenciando que variáveis componentes do bloco I (distal) perderam a significância após o ajuste final do modelo.

Ao incorporar as variáveis relacionadas às mães (bloco II - intermediário) observa-se que a DVA materna se associa à DVA dos filhos ( $\mathrm{p}=$ 0,001).

A relação inversa entre DVA e o peso ao nascer das crianças persistiu no modelo final ajustado, sugerindo que o peso ao nascer mais elevado seria um fator de proteção para a ocorrência da
DVA. Das cinco variáveis que compunham o bloco III (proximal), apenas o peso ao nascer $(p=0,04)$ e diarreia nos últimos quinze dias $(p=0,04)$ se mantiveram significativas no modelo final.

\section{Discussão}

Embora toda a população possa se achar exposta à DVA, são as crianças menores de cinco anos e as mulheres em idade reprodutiva, principalmente nos ciclos de gestação e lactação, que representam os segmentos biológicos mais vulneráveis à sua ocorrência ${ }^{1,2,4}$. É importante assina-

Tabela 2. continuação

\begin{tabular}{|c|c|c|c|c|c|c|c|c|}
\hline \multirow[b]{2}{*}{ Blocos/Variáveis } & \multicolumn{4}{|c|}{ Área urbana } & \multicolumn{4}{|c|}{ Área rural } \\
\hline & $\begin{array}{r}\text { Amostra } \\
\mathbf{n}=397^{*}\end{array}$ & DVA \% & RP (IC95\%) & $\mathrm{p}$ valor & $\begin{array}{l}\text { Amostra } \\
\mathrm{n}=393^{*}\end{array}$ & DVA\% & RP (IC95\%) & $\mathrm{p}$ valor \\
\hline \multicolumn{9}{|l|}{ Bloco II } \\
\hline \multicolumn{9}{|l|}{ Fatores maternos } \\
\hline Idade da mãe (anos) & & & & 0,02 & & & & 0,91 \\
\hline$<20$ & 31 & 29,0 & $2,13(1,16-3,90)$ & & 22 & 18,2 & $1,05(0,43-2,63)$ & \\
\hline$\geq 20$ & 366 & 13,7 & 1,0 & & 371 & 17,3 & 1,0 & \\
\hline DVA da mãe & & & & 0,45 & & & & 0,01 \\
\hline Sim & 25 & 8,0 & $0,60(0,16-2,35)$ & & 21 & 38,1 & $2,50(1,37-4,55)$ & \\
\hline Não & 325 & 13,2 & 1,0 & & 348 & 15,2 & 1,0 & \\
\hline Estado nutricional (IMC) & & & & 0,31 & & & & 0,31 \\
\hline Baixo peso & 21 & 9,5 & $0,49(0,08-2,34)$ & & 12 & 33,3 & $2,59(0,61-10,27)$ & \\
\hline Peso adequado & 198 & 17,7 & 1,0 & & 204 & 16,2 & 1,0 & \\
\hline Excesso de peso & 174 & 12,6 & $0,67(0,36-1,25)$ & & 175 & 17,1 & $1,07(0,60-1,91)$ & \\
\hline No consultas no pré-natal & & & & 0,02 & & & & 0,15 \\
\hline Não fez & 9 & 44,4 & $2,96(1,36-6,45)$ & & 13 & 7,7 & $0,39(0,06-2,60)$ & \\
\hline $1-5$ & 73 & 9,6 & $0,64(0,30-1,36)$ & & 120 & 12,5 & $0,63(0,37-1,08)$ & \\
\hline$\geq 6$ & 300 & 15,6 & 1,0 & & 243 & 19,8 & 1,0 & \\
\hline Suplementação Vitamina & & & & 0,59 & & & & 0,28 \\
\hline \multicolumn{9}{|l|}{ A no pós-parto } \\
\hline Sim & 23 & 21,7 & 1,0 & & 31 & 25,8 & 1,0 & \\
\hline Não & 45 & 13,3 & $0,61(0,21-1,80)$ & & 41 & 22,0 & $0,85(0,37-1,95)$ & \\
\hline $\begin{array}{l}\text { Não teve filhos nos } \\
\text { últimos } 12 \text { meses }\end{array}$ & 325 & 14,2 & $0,65(0,29-1,48)$ & & 319 & 16,0 & $0,62(0,32-1,18)$ & \\
\hline \multicolumn{9}{|l|}{$\begin{array}{l}\text { Acesso a serviços de saúde/ } \\
\text { programas sociais }\end{array}$} \\
\hline Visita do agente de saúde & & & & 0,79 & & & & 0,84 \\
\hline Sim (PSF/PACS) & 311 & 15,1 & 1,0 & & 343 & 17,5 & 1,0 & \\
\hline Não & 86 & 14,0 & $0,92(0,51-1,66)$ & & 49 & 16,3 & $0,93(0,48-1,83)$ & \\
\hline Cadastro no PSF & & & & 0,75 & & & & 0,51 \\
\hline Sim & 223 & 14,3 & 1,0 & & 276 & 18,1 & 1,0 & \\
\hline Não & 174 & 15,5 & $1,08(0,67-1,73)$ & & 117 & 15,4 & $0,85(05,2-1,39)$ & \\
\hline Inscrição no PBF & & & & 0,82 & & & & 0,89 \\
\hline Sim & 264 & 15,2 & 1,0 & & 333 & 17,4 & 1,0 & \\
\hline Não & 133 & 14,3 & $0,94(0,57-1,56)$ & & 60 & 16,7 & $0,96(05,2-1,77)$ & \\
\hline
\end{tabular}


lar que a DVA, como todos os problemas carenciais de interesse epidemiológico, apresenta um evidente viés socioeconômico, prevalecendo nos países, regiões e famílias mais desfavorecidas na estratificação das desigualdades de renda, educação, moradia, acesso aos serviços de saúde e outros aspectos, como o saneamento da habitação. Nesse contexto de adversidades, a condição econômica exerce um papel crucial, justificando o critério da OMS em reunir e sistematizar informações sobre a situação da vitamina $\mathrm{A}$, para o período de 1995 a 2005, dos países com renda per capita abaixo de 15 mil dólares ${ }^{1}$. É esta, ainda, a situação do Brasil e, mais ainda, do Estado de Pernambuco, com uma renda per capita estimada em 3050 dólares para o ano de $2006^{19}$, quan-

Tabela 2. continuação

\begin{tabular}{|c|c|c|c|c|c|c|c|c|}
\hline \multirow[b]{2}{*}{ Blocos/Variáveis } & \multicolumn{4}{|c|}{ Área urbana } & \multicolumn{4}{|c|}{ Área rural } \\
\hline & $\begin{array}{r}\text { Amostra } \\
\mathbf{n}=397^{*}\end{array}$ & DVA \% & RP (IC95\%) & $\mathrm{p}$ valor & $\begin{array}{c}\text { Amostra } \\
\mathrm{n}=393^{*}\end{array}$ & DVA\% & RP (IC95\%) & $\mathrm{p}$ valor \\
\hline \multicolumn{9}{|l|}{ Bloco III } \\
\hline \multicolumn{9}{|l|}{ Fatores relacionados à criança } \\
\hline \multicolumn{9}{|l|}{ Biológicos } \\
\hline Sexo & & & & 0,58 & & & & 0,53 \\
\hline Masculino & 202 & 15,8 & $1,14(0,71-1,84)$ & & 206 & 18,4 & $1,15(0,74-1,78)$ & \\
\hline Feminino & 195 & 13,8 & 1,0 & & 187 & 16,0 & 1,0 & \\
\hline Idade (meses) & & & & 0,22 & & & & 0,11 \\
\hline$<24$ & 134 & 17,9 & $1,35(0,84-2,17)$ & & 124 & 21,8 & $1,43(0,92-2,21)$ & \\
\hline$\geq 24$ & 263 & 13,3 & 1,0 & & 269 & 15,2 & 1,0 & \\
\hline Peso ao nascer (g) & & & & 0,06 & & & & 0,002 \\
\hline$<2500$ & 30 & 10,0 & $0,57(0,19-1,73)$ & & 26 & 42,3 & $2,78(1,65-4,68)$ & \\
\hline $2500 \vdash 3000$ & 80 & 7,5 & $0,43(0,19-0,97)$ & & 65 & 16,9 & $1,11(0,61-2,03)$ & \\
\hline$\geq 3000$ & 287 & 17,4 & 1,0 & & 302 & 15,2 & 1,0 & \\
\hline $\begin{array}{l}\text { Estado nutricional } \\
\text { (Peso/idade) }\end{array}$ & \multicolumn{7}{|c|}{ Estado nutricional } & 0,16 \\
\hline Baixo peso & 9 & 22,2 & $1,57(0,45-5,47)$ & & 11 & 36,4 & $2,14(0,95-4,82)$ & \\
\hline Peso normal & 353 & 14,2 & 1,0 & & 369 & 17,1 & 1,0 & \\
\hline Peso elevado & 24 & 16,7 & $1,18(0,46-2,98)$ & & 13 & 7,7 & $0,45(0,07-2,99)$ & \\
\hline (Estatura/idade) & & & & 0,95 & & & & 0,16 \\
\hline Baixa estatura & 20 & 15,0 & $1,04(0,35-3,03)$ & & 49 & 24,5 & $1,50(0,87-2,60)$ & \\
\hline Estatura adequada & 366 & 14,5 & 1,0 & & 344 & 16,3 & 1,0 & \\
\hline \multicolumn{9}{|l|}{ Alimentação } \\
\hline Amamentação (meses & & & & 0,52 & & & & 0,29 \\
\hline Nunca mamou & 68 & 16,2 & $0,98(0,52-1,83)$ & & 76 & 15,8 & $1,08(0,58-2,01)$ & \\
\hline $1-2$ & 66 & 9,1 & $0,55(0,24-1,26)$ & & 69 & 18,8 & $1,28(0,70-2,34)$ & \\
\hline $3-4$ & 76 & 14,5 & $0,87(0,46-1,65)$ & & 64 & 25,0 & $1,70(0,98-2,95)$ & \\
\hline$\geq 5$ & 187 & 16,6 & 1,0 & & 184 & 14,7 & 1,0 & \\
\hline $\begin{array}{l}\text { Suplementação Vit. A } \\
\text { (últimos } 6 \text { meses) }\end{array}$ & & & & 0,80 & & & & 0,73 \\
\hline Sim & 207 & 15,5 & 1,0 & & 164 & 16,5 & 1,0 & \\
\hline Não & 179 & 14,5 & $0,94(0,58-1,51)$ & & 219 & 17,8 & $1,08(0,69-1,69)$ & \\
\hline \multicolumn{9}{|l|}{ Morbidades } \\
\hline Diarreia nos últimos & & & & 0,11 & & & & 0,02 \\
\hline \multicolumn{9}{|l|}{15 dias } \\
\hline Sim & 60 & 21,7 & $1,59(0,91-2,75)$ & & 71 & 26,8 & $1,75(1,11-2,79)$ & \\
\hline Não & 337 & 13,6 & 1,0 & & 322 & 15,2 & 1,0 & \\
\hline Anemia (hemoglobina & & & & 0,33 & & & & 0,84 \\
\hline $\operatorname{Sim}(<11 \mathrm{~g} / \mathrm{dL})$ & 115 & 17,4 & $1,20(0,70-2,10)$ & & 137 & 16,8 & $0,96(0,60-1,51)$ & \\
\hline Não $(\geq 11 \mathrm{~g} / \mathrm{dL})$ & 280 & 13,6 & 1,0 & & 256 & 17,6 & 1,0 & \\
\hline
\end{tabular}

"Os totais parciais de cada grupo podem diferir do total da amostra em função de informações ignoradas pelas entrevistadas. "*Salário mínimo na época: $\mathrm{R} \$ 350,00$ 
do foi realizada a pesquisa aqui apresentada. No relatório da $\mathrm{OMS}^{1}$ revelou-se que no conjunto dos 51 países analisados, 33,3\% das crianças e $15,3 \%$ das gestantes do mundo em desenvolvimento apresentavam níveis de retinol sérico abaixo de $0,70 \mu \mathrm{mol} / \mathrm{L}$ ( ou $20 \mu \mathrm{g} / \mathrm{dL}$ ) caracterizando situações onde a DVA constitui um evidente problema de saúde pública.

No caso do Brasil, dados da Pesquisa Nacional de Demografia e Saúde (PNDS/2006) ${ }^{8}$ realizada no mesmo ano do inquérito de Pernambuco, revelaram um cenário de certa forma inesperado, já que, a região mais rica, o Sudeste, destaca-se com as ocorrências mais elevadas em crianças $(21,6 \%)$ e mulheres em idade reprodutiva (14\%), seguindo-se do Nordeste brasileiro. Relata-se ainda que a DVA atingia todas as regiões do Brasil ${ }^{8}$, contrariando a impressão dominante até então de que o problema estaria limitado ao Norte e Nordeste e, excepcionalmente, as microrregiões de pobreza, como o Vale do Jequitinhonha em Minas Gerais e o Vale do Ribeira em São Paulo ${ }^{6,7}$.

Na realidade, a prevalência de DVA em 16,1\% das crianças e 6,9\% das mães em Pernambuco, em 2006, seria aceitável e até previsível, considerando os resultados obtidos em menores de cinco anos neste Estado na avaliação de $1997^{17}$. No entanto, torna-se, inesperada nas mulheres quando comparado aos resultados da PNDS $/ 2006^{8}$ em nível nacional e macrorregional. Detalhan- do-se: entre as crianças, os resultados obtidos em Pernambuco $(16,1 \%)$ são plenamente compatíveis com dados anteriores do próprio Estado $^{17}(18,8 \%$, em 1997) e com o vizinho estado da Paraíba $^{7}$ (16\%). Convém lembrar que desde 1983 o controle da DVA foi implantado (mas não efetivamente sistematizado no Brasil) sendo o estado de Pernambuco uma das unidades federativas onde as ações de intervenções mais se desenvolveram ${ }^{7}$. Já entre as mulheres $(6,9 \%)$ divergem significativamente dos resultados da PNDS $/ 2006^{8}$ em nível nacional (12,3\%) e regional com $12,1 \%$ das do Nordeste, ou seja, quase duas vezes mais elevada. Em razão das diferenças de prevalência, destaca-se que, em Pernambuco, a DVA seria uma condição epidemiológica classificada ${ }^{1}$ como leve para as mulheres, enquanto os dados do inquérito nacional ${ }^{8}$ colocariam o problema na categoria de moderado. Já para os menores de cinco anos, a condição de problema moderado em Pernambuco se assemelha à classificação da PNDS/ $2006^{8}$ para o país.

Além da pesquisa nacional ${ }^{8}$, outros estudos de nível local ou estadual descrevem situações bem mais graves em crianças: 32,1\% em Sergipe $^{20}$ e $44,8 \%$ na zona semiárida de Alagoas ${ }^{21}$. Ademais, a situação do meio rural de Pernambuco, contrariando os poucos estudos produzidos em relação à dicotomia campo/cidade, denota uma mudança que tende a favorecer as cri-

Tabela 3. Prevalência da deficiência de vitamina A (DVA) e de razão de prevalência (RP) bruta e ajustada para crianças menores de cinco anos residentes na área urbana. Pernambuco, 2006.

\begin{tabular}{|c|c|c|c|c|c|}
\hline \multirow[b]{2}{*}{ Blocos/Variáveis } & \multicolumn{5}{|c|}{ Área urbana } \\
\hline & $\begin{array}{c}\text { DVA } \\
\%\end{array}$ & $\begin{array}{l}\mathbf{R P}_{\text {bruta }} \\
\text { (IC 95\%) }\end{array}$ & $\mathrm{p}$ valor & $\begin{array}{l}\mathbf{R P}_{\text {ajustada }} \\
\text { (IC 95\%) }\end{array}$ & $\begin{array}{c}\mathrm{p} \\
\text { valor }\end{array}$ \\
\hline \multicolumn{6}{|l|}{ Bloco II } \\
\hline Idade da mãe (anos) & & & 0,02 & & 0,004 \\
\hline$<20$ & 29,0 & $2,13(1,16-3,90)$ & & $3,00(1,43-6,31)$ & \\
\hline$\geq 20$ & 13,7 & 1,0 & & 1,0 & \\
\hline No de consultas no pré-natal & & & 0,02 & & 0,006 \\
\hline Não fez & 44,4 & $2,96(1,36-6,45)$ & & $3,99(1,41-11,30)$ & \\
\hline $1-5$ & 9,6 & $0,64(0,30-1,36)$ & & $0,51(0,22-1,15)$ & \\
\hline$\geq 6$ & 15,6 & 1,0 & & 1,0 & \\
\hline \multicolumn{6}{|l|}{ Bloco III } \\
\hline Peso ano nascer (g) & & & 0,06 & & 0,01 \\
\hline$<2500$ & 10,0 & $0,57(0,19-1,73)$ & & $0,26(0,06-1,13)$ & \\
\hline $2500 \vdash 3000$ & 7,5 & $0,43(0,19-0,97)$ & & $0,32(0,13-0,80)$ & \\
\hline$\geq 3000$ & 17,4 & 1,0 & & 1,0 & \\
\hline Diarreia nos últimos quinze dias & & & 0,11 & & 0,12 \\
\hline Sim & 21,7 & $1,59(0,91-2,75)$ & & $1,66(0,87-3,15)$ & \\
\hline Não & 13,6 & 1,0 & & 1,0 & \\
\hline
\end{tabular}


anças de famílias camponesas lamentando-se o fato de não se dispor de informações no passado que permitam estabelecer comparações em relação às mulheres. A mudança da situação entre as crianças do meio rural é um resultado inesperado, tendo em vista o cenário adverso que se desenhava sistematicamente em relação a este, no Brasil $^{22}$ e no mundo ${ }^{23,24}$. Pode, de fato, revelar a emergência de uma nova situação. Pesquisadores $^{24}$ que avaliaram dados de 47 países em desenvolvimento evidenciaram que a lacuna entre o espaço urbano e o rural tende a diminuir, levando os autores a questionar se as crianças nas cidades seriam atualmente mais saudáveis do que as que residem no campo. No aspecto nutricional, este questionamento também se aplicaria ao caso de Pernambuco e do próprio Brasil ${ }^{8}$, revelando um aspecto novo da transição epidemiológica que estamos atravessando.

$\mathrm{Na}$ grande maioria dos estudos evidencia-se que os riscos de DVA são bem mais elevados nas crianças do que entre mulheres em idade fértil,

Tabela 4. Prevalência da deficiência de vitamina A (DVA) e de razão de prevalência (RP) bruta e ajustada para crianças menores de cinco anos residentes na área rural. Pernambuco, 2006.

\begin{tabular}{|c|c|c|c|c|c|}
\hline \multirow[b]{2}{*}{ Variável (nível) } & \multicolumn{5}{|c|}{ Área rural } \\
\hline & $\begin{array}{c}\text { DVA } \\
\%\end{array}$ & $\begin{array}{l}\mathrm{RP}_{\text {bruta }} \\
(\mathrm{IC} \mathbf{9 5 \%})\end{array}$ & $\mathrm{p}$ valor & $\begin{array}{l}\mathrm{RP}_{\text {ajustada }} \\
\text { (IC 95\%) }\end{array}$ & p valor \\
\hline \multicolumn{6}{|l|}{ Bloco I } \\
\hline Escolaridade materna (anos) & & & 0,14 & & 0,14 \\
\hline 0 & 21,4 & $0,72(0,32-1,64)$ & & $0,68(0,26-1,78)$ & \\
\hline $1-8$ & 15,8 & $0,53(0,28-1,00)$ & & $0,49(0,23-1,04)$ & \\
\hline$\geq 9$ & 29,6 & 1,0 & & 1,0 & \\
\hline Regime de ocupação & & & 0,06 & & 0,08 \\
\hline Casa própria & 15,3 & 1,0 & & 1,0 & \\
\hline $\begin{array}{l}\text { Casa alugada/cedida/invadida/ } \\
\text { outro }\end{array}$ & 23,9 & $1,56(1,00-2,46)$ & & $1,59(0,95-2,66)$ & \\
\hline Destino do lixo & & & 0,03 & & 0,06 \\
\hline Coleta pública & 7,0 & 1,0 & & 1,0 & \\
\hline Outra forma & 19,0 & $2,71(1,03-7,16)$ & & $2,70(0,98-7,42)$ & \\
\hline \multicolumn{6}{|l|}{ Bloco II } \\
\hline DVA da mãe (retinol sérico) & & & 0,006 & & 0,001 \\
\hline Deficiente $(<20 \mu \mathrm{g} / \mathrm{dL})$ & 38,1 & $2,50(1,37-4,55)$ & & $3,99(1,73-9,19)$ & \\
\hline Normal $(\geq 20 \mu \mathrm{g} / \mathrm{dL})$ & 15,2 & 1,0 & & 1,0 & \\
\hline No consultas no pré-natal & & & 0,15 & & 0,11 \\
\hline Não fez & 7,7 & $0,39(0,06-2,60)$ & & $0,25(0,03-1,93)$ & \\
\hline $1-5$ & 12,5 & $0,63(0,37-1,08)$ & & $0,58(0,31-1,07)$ & \\
\hline$\geq 6$ & 19,8 & 1,0 & & 1,0 & \\
\hline \multicolumn{6}{|l|}{ Bloco III } \\
\hline Idade (meses) & & & 0,11 & & 0,17 \\
\hline$<24$ & 21,8 & $1,43(0,92-2,21)$ & & $1,46(0,85-2,50)$ & \\
\hline$\geq 24$ & 15,2 & 1,0 & & 1,0 & \\
\hline Peso ao nascer $(\mathrm{g})$ & & & 0,002 & & 0,04 \\
\hline$<2500$ & 42,3 & $2,78(1,65-4,68)$ & & $2,80(1,22-6,42)$ & \\
\hline $2500 \vdash 3000$ & 16,9 & $1,11(0,61-2,03)$ & & $0,85(0,42-1,75)$ & \\
\hline$\geq 3000$ & 15,2 & 1,0 & & 1,0 & \\
\hline Diarreia nos últimos quinze dias & & & 0,02 & & 0,04 \\
\hline $\operatorname{Sim}$ & 26,8 & $1,75(1,11-2,79)$ & & $1,88(1,04-3,41)$ & \\
\hline Não & 15,2 & 1,0 & & 1,0 & \\
\hline Estado nutricional (Peso/idade) & & & 0,16 & & 0,35 \\
\hline Baixo peso & 36,4 & $2,14(0,95-4,82)$ & & $1,98(0,64-6,17)$ & \\
\hline Peso normal & 17,1 & 1,0 & & 1,0 & \\
\hline Peso elevado & 7,7 & $0,45(0,07-2,99)$ & & $0,49(0,07-3,55)$ & \\
\hline Estado nutricional (Estatura/idade) & & & 0,16 & & 0,95 \\
\hline Baixa estatura & 24,5 & $1,50(0,87-2,60)$ & & $1,03(0,48-2,16)$ & \\
\hline Estatura adequada & 16,3 & 1,0 & & 1,0 & \\
\hline
\end{tabular}


em termos de indicadores bioquímicos ${ }^{1,23}$. Já em relação aos sinais e sintomas oculares a situação se inverte ${ }^{1,4}$, sendo as ocorrências mais comuns em mulheres em idade reprodutiva. Segundo a publicação da $\mathrm{OMS}^{1}$, a razão de prevalência da DVA no mundo entre crianças $(33,3 \%)$ e mulheres $(15,3 \%)$ é de 2,2. Recorrendo-se à mesma comparação, a razão encontrada em Pernambuco seria praticamente a mesma (2,3 vezes), decaindo para 1,4 vezes para o Brasil $^{8}$ em seu conjunto. No entanto, a originalidade do inquérito de Pernambuco consiste no pareamento mãe-filho biológico, constituindo um binômio que, pertencendo à mesma família, participa de um micro-ambiente comum (renda, habitação, hábitos de consumo, inclusive de alimentos, valores culturais, acesso às ações de saúde e outras condições correlatas). Hipoteticamente, portanto, estas condições deveriam reunir fatores de risco também semelhantes.

No caso de Pernambuco, isso só ocorre parcialmente. Em princípio, as diferenças de prevalências e o enquadramento também diferenciado da classificação epidemiológica do problema (leve em mães, moderado nas crianças) já apontam intensidades diversas, é possível admitir que os fatores significativos para sua determinação possam ser também distintos, em termos de natureza qualitativa. É esta a perspectiva preliminar para se analisar, ao lado das prevalências, a associação isolada e/ou combinada de fatores hipoteticamente causais da DVA em mães e filhos. Assim, nas análises bivariadas, uma das implicações práticas foi a constatação de que a prevalência inesperadamente baixa do problema nas mães resultou na dificuldade de se encontrar associações estatísticas com as variáveis de possível interesse para compor o modelo explicativo. Dessa forma, apenas numa situação (regime de ocupação da moradia na área urbana) os resultados de DVA foram demonstrativos de diferenças estatisticamente significativas entre as mães. É um resultado curioso, não ocorrendo uma explicação devidamente fundamentada.

Portanto, o que de fato passa a ser interessante, são os fatores que se associaram a DVA em crianças. Nesta condição, é pertinente considerar que a deficiência de vitamina A da mãe passa a ser, agora analisada como condição de risco para a criança, ou seja, uma variável associada à DVA dos filhos. Esta associação estatística, que se evidencia no meio rural, se mantém, afinal, no modelo hierárquico após os devidos ajustamentos. Este é um aspecto muito singular da presente pesquisa, uma vez que outro estudo, efetuado no Sul da África ${ }^{25}$ numa aldeia caracterizada por elevada prevalência de desnutrição, anemia e DVA em crianças, não encontrou associação entre a DVA das mães e de seus filhos, situação semelhante à encontrada no meio urbano de Pernambuco.

Fora do plano epidemiológico, existem estudos explorando relações basicamente clínicas, como o retinol sérico de mães e do cordão umbilical dos recém-nascidos ${ }^{26}$ ou a revisão sistemática de Caminha et al. ${ }^{27}$ sobre o conteúdo de vitamina A no leite materno disponibilizado para lactentes. Embora importante como contribuição sobre a fisiopatologia da nutrição na vida intrauterina e seu possível impacto nas relações nutricionais entre mães e filhos, essas abordagens não esclarecem a situação nos meses e anos de vida que se seguem após o nascimento, quando, de fato, se instala o maior risco de DVA.

Estudos efetuados no Brasil nos últimos 10 anos são pouco conclusivos ou pouco convincentes, no sentido de identificar fatores associados à DVA de crianças. A renda da família, por exemplo, se associa aos riscos de deficiência de vitamina A na II PESN -PE/1997' ${ }^{17}$, e ainda em estudos em Sergipe ${ }^{20}$ e em Gameleira ${ }^{28}$, no estado de Pernambuco. No entanto, essa associação não foi encontrada no nosso estudo, no estrato urbano e rural, na PNDS $/ 2006^{8}$ e em São João do Tigre, estado da Paraíba ${ }^{28}$. Chama a atenção ainda, a não associação entre a DVA e a baixa escolaridade materna (quase consensual em pesquisas nacionais e internacionais).

Nas crianças do meio urbano, a idade da mãe inferior a 20 anos associou-se significativamente com a ocorrência de DVA ( $p=0,004)$, enquanto no meio rural, as diferenças não foram significantes. No estado de Sergipe ${ }^{20}$, apesar da ocorrência do problema ser mais elevado nos filhos cujas mães eram menores de 20 anos, não foi encontrada significância estatística entre idade materna e DVA dos filhos.

A não realização de consultas no pré-natal foi um fator significativo de risco de DVA nos filhos de mães residentes no meio urbano. Contrariamente, o mesmo não aconteceu com as crianças e as mães que residem no meio rural, as frequências mais baixas de DVA, apesar da não significância estatística, foram encontradas no pequeno grupo de mães sem nenhuma consulta. Andrade $^{17}$ identificou uma situação semelhante ao analisar a DVA em crianças cujas mães não realizaram consultas no pré-natal no estado de Pernambuco.

O baixo peso ao nascer $(<2500 \mathrm{~g})$ se apresenta como um fator de risco de DVA nas crianças 
em áreas rurais, enquanto que no meio urbano essa associação é significante nas crianças maiores de 3000g. Não há lógica aparente para esta contradição de resultados no contexto do estudo nem achados na literatura para sua explicação. Somente um estudo anterior realizado no estado de Pernambuco ${ }^{17}$ o qual não encontrou associação entre baixo peso ao nascer e DVA.

Em nosso estudo, não foi encontrada a associação estatisticamente significativa entre indicadores antropométricos (P/I e A/I) e a ocorrência de DVA em crianças. Em Pernambuco com dados do inquérito efetuado em 1997, Andrade ${ }^{17}$ também não encontrou associação entre a DVA e o déficit antropométrico pela relação peso/idade. Já Martins et al. ${ }^{20}$ analisando dados da pesquisa estadual de Sergipe observaram que a distribuição de retinol sérico se mostrou associada ao indicador peso/idade. Teixeira ${ }^{29}$ avaliando dois municípios do semiárido de Minas Gerais, concluiu que as crianças com déficit de estatura, em Francisco Badaró, tinham um risco 5,6 vezes maior de apresentar DVA.

Ao contrário do que tem sido observado em alguns estudos ${ }^{25,30,31}$, não foi encontrada associação entre anemia e DVA em crianças no estado de Pernambuco. Na realidade, parece que as relações entre anemia e DVA em crianças dependem de contextos epidemiológicos próprios de cada processo carencial isolado ou combinado, como ocorre nos diversos ecossistemas afetados pelos problemas de nutrição.

Por fim, uma constatação esperada: a associação da DVA nas crianças com a ocorrência de diarreia nos últimos 15 dias. Ressalvada a limitação preliminar de que os estudos de prevalência registrariam causa e efeitos simultâneos, portanto, sem estabelecer a temporalidade, é pertinente observar que a DVA é, reconhecidamente, um fator de risco para as diarreias infecciosas ${ }^{5,32,33} \mathrm{e}$ que as diarreias podem precipitar ou agravar o processo carencial de vitamina $\mathrm{A}^{2,4}$. Esta relação evidenciada nas crianças rurais, provavelmente seria menos comum nas crianças do meio urbano, onde as condições de saneamento das famílias são bem melhores.

Pelo exposto, subsistem vários pontos a serem esclarecidos referentes à compreensão da DVA. A adoção de novos pontos de corte para fundamentar as classificações epidemiológicas a partir de $1996^{12}$, questões básicas de amostragem (com a predominância de estudos pontualmente limitados no espaço geográfico), a falta de indicadores sobre o consumo de alimentos. Além destes, outros fatores como a eleição de um gran- de número de variáveis independentes e seus critérios de estratificação e análise, a possível estacionalidade da DVA em alguns ecossistemas (semiárido e áreas de monoculturas) e até mesmo de validação de indicadores, nas condições ambientais e logísticas onde as pesquisas são aplicadas. No caso de Pernambuco, na medida em que se utilizou a mesma metodologia de amostragem, praticamente a mesma equipe de coleta de dados de campo e os mesmos procedimentos laboratoriais usados na pesquisa estadual em $1997^{17}$, grande parte destes questionamentos poderiam ser, de certa maneira, minimizados em termos de comparabilidade. Cabe destacar ainda, uma limitação importante: a não disponibilidade de dados de mães e seus filhos sobre o consumo de alimentos fontes de vitamina A como um elemento chave para se entender o fator primordial para a gênese do problema da DVA.

Em comparação com os resultados do Brasil e do Nordeste, as prevalências obtidas em Pernambuco são bem menores que os valores descritos pela $\mathrm{PNDS} / 2006^{8}$, no que se refere às mulheres em idade reprodutiva, sem diferir significativamente na comparação com as crianças. As diferenças históricas, quase consensuais, desfavorecendo sistematicamente mães e crianças do meio rural em relação ao ambiente urbano, foram substancialmente modificadas ${ }^{8}$, tornandose praticamente equivalentes. Estas diferenças temporais, geográficas, biológicas e sociais evidenciadas na análise da DVA em crianças e mães no estado de Pernambuco e suas comparações com outros estudos, põem em foco a justificativa de se rever as agendas de pesquisas, fundamentos, estratégias e metas de políticas públicas, programas e ações propostos para o enfrentamento da DVA no estado e, muito provavelmente, em toda a região.

\section{Colaboradores}

TC Miglioli participou da pesquisa bibliográfica, processamento e análise de dados e redação do texto. VM Fonseca trabalhou na concepção e coordenação da análise dos dados, redação e revisão final do texto. SC Gomes Junior participou da análise estatística e revisão final do texto. PIC Lira contribuiu na coordenação do projeto, redação e revisão final do texto. M Batista Filho participou da concepção e coordenação do projeto, redação e revisão final do texto. 


\section{Agradecimentos}

Ao Conselho Nacional de Desenvolvimento Científico e Tecnológico (CNPq) pelo financiamento da pesquisa: Situação alimentar nutricional e de saúde no Estado de Pernambuco: contexto socioeconômico e de serviços. A Coordenação de Pessoal de Nível Superior (Capes) pela bolsa de doutorado de Teresa Cristina Miglioli e ao $\mathrm{CNPq}$ pelas bolsas de produtividade em pesquisa de Malaquias Batista Filho e Pedro Israel Cabral de Lira.

\section{Referências}

1. World Health Organization (WHO). Global prevalence of vitamin A deficiency in populations at risk 1995-2005. WHO Global Database on Vitamin A Deficiency. Geneve: WHO; 2009.

2. Ramalho A, Dolinsky M. Carência de vitamina A no grupo materno-infantil. In: Accioly E, Saunders C, Lacerda EMA, organizadores. Nutrição em obstetrícia e pediatria. 2a Edição. Rio de Janeiro: Cultura Médica, Guanabara Koogan; 2009. p. 57-75.

3. World Health Organization (WHO). Iron deficiency anaemia: assessment, prevention and control. A guide for programme managers. Geneve: WHO; 2001.

4. West Junior KP. Vitamin A deficiency disorders in children and women. Food Nutr Bull 2003; 24(Supl. 4):78-93.

5. Mayo-Wilson E, Imdad A, Herzer K, Yakoob MY, Bhutta ZA. Vitamin A supplements for preventing mortality, illness, and blindness in children aged under 5: systematic review and meta-analysis. BMJ 2011; 343(7833):d5094.

6. Milagres RCRM, Nunes LC, Pinheiro-Sant'Ana HM. A deficiência de vitamina A em crianças no Brasil e no mundo. Cienc Saude Colet 2007; 12(5):1253-1266.

7. Martins MC, Oliveira YP, Coitinho DC, Santos LMP. Panorama das ações de controle da deficiência de vitamina A no Brasil. Rev Nutr Campinas 2007; 20(1):5-18.

8. Brasil. Ministério da Saúde (MS). Pesquisa Nacional de Demografia e Saúde da Criança e da MulherPNDS 2006: dimensões do processo reprodutivo e da saúde da criança. Brasília: MS; 2009. [acessado 2013 mar 28]. Disponível em: http://bvsms.saude. gov.br/bvs/publicacoes/pnds_crianca_mulher.pdf

9. Brasil. Ministério da Saúde (MS). Saúde da família: uma estratégia para a reorientação do modelo assistencial. Brasília: MS; 1997.

10. Miglioli TC, Brito AM, Lira PIC, Figueroa JN, Batista Filho M. Anemia no binômio mãe-filho no Estado de Pernambuco, Brasil. Cad Saude Publica 2010; 26(9):1807-1820

11. Furr HC, Tanumihardjo SA, Olson JA. Training manual for assessing vitamin A status by use of the modified relative dose response and the relative dose response assays. Washington (DC): Sponsored by the USAID Vitamin A Field Support Project-VITAL.; 1992.

12. World Health Organization (WHO). Indicators for assessing vitamin $A$ deficiency and their application in monitoring and evaluating intervention programmes. Geneve: WHO; 1996.

13. World Health Organization (WHO). Anthro for personal computers. Version 2. 2007: Software for assessing growth and development of the world's children. Geneva: WHO; 2007. [acessado 2013 mar 28]. Disponível em: http://www.who.int/childgrowth/ software/en/

14. World Health Organization (WHO). WHO Child Growth Standards: Length/height-for-age, weightforage, weight-for-length, weight-for-height and body mass index-for-age. Methods and development. Geneva: WHO; 2006. 
15. World Health Organization (WHO). The international classification of adult underweight, overweight and obesity according to BMI. Geneve: WHO; 2004.

16. Onis M, Onyango AW, Borghi E, Siyam A, Nishida C, Siekmann J. Development of a WHO growth reference for school-aged children and adolescents. Bull World Health Organ 2007; 85(9):660-667.

17. Andrade SLLS. Prevalência de hipovitaminose A e potenciais fatores de risco em crianças menores de 5 anos no estado de Pernambuco 1997 [tese]. Recife: Departamento de Nutrição da Universidade Federal de Pernambuco; 2000.

18. Penman AD, Johnson WD. Complementary LogLog Regression for the Estimation of CovariateAdjusted Prevalence Ratios in the Analysis of Data from Cross-Sectional Studies. Biometrical J 2009; 51(3):433-442.

19. Instituto Brasileiro de Geografia e Estatística (IBGE). Indicadores. Estatísticas. Rio de Janeiro: IBGE. [acessado em 2013 mar 28]. Disponível em: http:// www.ibge.gov.br/home/download/estatistica.shtm

20. Martins MC, Santos LMP, Assis AMO. Prevalência da hipovitaminose A em pré-escolares no Estado de Sergipe, 1998. Rev Saude Publica 2004; 38(4):537542.

21. Vasconcelos AMA, Ferreira HS. Prevalência de hipovitaminose $\mathrm{A}$ em crianças da região semi-árida de Alagoas (Brasil), 2007. Arch Latinoam Nutr 2009; 59(2):152-158.

22. Santos LMP, organizador. Bibliografia sobre deficiência de micronutrientes no Brasil 1990-2000: volume 1 vitamina A. Brasília: Organização Pan-Americana da Saúde; 2002.

23. Dommarco JR, Levy TS, SV Hernández, Cossío TG, Prado BH, Sepúlveda J. Encuesta Nacional de Nutrición 1999. Estado nutrício de niños y mujeres en México. Cuernavaca, Morelos, México: Instituto Nacional de Salud Pública; 2001. [acessado 2013 mar 28]. Disponível em: http://www.salud.gob.mx/ unidades/cdi/documentos/nutricion.pdf

24. Van de Poel E, O’Donnell O, Van Doorslaer E. Are urban children really healthier? Evidence from 47 developing countries. Soc Sci Med 2007; 65(10):19862003.

25. Faber M, Swanevelder S, Benadé AJ. Is there an association between the nutritional status of the mother and that of her 2-year-old to 5-year-old child? Int J Food Sci Nutr 2005; 56(4):237-244.
26. Ramalho RA, Flores H, Accioly E, Saunder C. Associação entre deficiência de vitamina $\mathrm{A}$ e situação sociodemográfica de mães e recém-nascidos. Rev Assoc Med Bras 2006; 52(3):170-175.

27. Caminha MFC, Batista Filho M, Fernandes TFS, Arruda IKG, Diniz AS. Suplementação com vitamina A no puerpério: revisão sistemática. Rev Saude Publica 2009; 43(4):699-706.

28. Oliveira JS, Lira PIC, Osório MM, Sequeira LAS, Costa EC, Gonçalves FCLSP, Batista Filho M. Anemia, hipovitaminose A e insegurança alimentar em crianças de municípios de Baixo Índice de Desenvolvimento Humano do Nordeste do Brasil. Rev Bras Epidemiol 2010; 13(4):651-664.

29. Teixeira RA. Deficiência de vitamina A e fatores associados em crianças e adolescentes em dois municípios do semiárido de Minas Gerais [tese]. Belo Horizonte: Universidade Federal de Minas Gerais; 2010.

30. Chen K, Zhang X, Li TY, Chen L, Qu P, Liu YX. Coassessment of iron, vitamin $\mathrm{A}$ and growth status to investigate anemia in preschool children in suburb Chongqing, China. World J Pediatr 2009; 5(4):275281.

31. Silva RCR, Assis AMO, Santana MLP, Barreto ML, Brito LL, Reis MG, Parraga IM, Blanton RE. Relação entre os níveis de vitamina $\mathrm{A}$ e os marcadores bioquímicos do estado nutricional de ferro em crianças e adolescentes. Rev Nutr 2008; 21(3):285-291.

32. Geraldo RRC, Paiva SAR, Pitas AMCS, Godoy I, Campana AO. Distribuição da hipovitaminose A no Brasil nas últimas quatro décadas: ingestão alimentar, sinais clínicos e dados bioquímicos. Rev Nutr 2003; 16(4):443-460.

33. Oliveira JM, Rondó PHC. Evidências do impacto da suplementação de vitamina A no grupo materno-infantil. Cad Saude Publica 2007; 23(11):25652575.

Artigo apresentado em 02/03/2012

Aprovado em 25/04/2012

Versão final apresentada em 07/05/2012 